\title{
Aplicaciones y retos del sensado remoto hiperespectral en la geología colombiona
}

\author{
Applications and challenges of hyperspectral remote sensing in \\ the colombian geology
}

\section{Aplicações e desafios do sensoriamento remoto hiperespectral na geologia colombiana}

Fecha Recepción: 10 de Febrero de 2015

Fecha Aprobación: 16 de Junio de 2015

\author{
Ariolfo Camacho-Velasco* \\ Cesar Augusto Vargas-García* \\ Fernando Antonio Rojas-Morales ${ }^{* * *}$ \\ Sergio Fernando Castillo-Castelblanco ${ }^{\text {*tot }}$ \\ Henry Arguello-Fuentes
}

\section{Resumen}

El Sensado Remoto (SR) es una técnica que permite captar información de una escena sin entrar en contacto físico con ella, mediante el empleo de sensores ubicados, principalmente, en plataformas aéreas, los cuales captan información en diferentes rangos del espectro electromagnético, incluyendo el visible (VIS), el cercano al infrarrojo (NIR) y el de ondas cortas del infrarrojo (SWIR). Teniendo en cuenta que cada material presente en una escena tiene características espectrales diferentes, es posible, a través del análisis de las firmas espectrales, realizar su identificación o clasificación mediante algoritmos. Las Imágenes Hiperespectrales (HSI) captadas por sensores remotos en cientos de bandas espectrales son de importancia en áreas como la geología, la mineralogía, la agronomía y la ecología, entre otras; sin embargo, el gran volumen de literatura dispersa en diferentes líneas (SR, HSI y geología) dificulta su acceso y análisis. Este trabajo presenta un compendio de conceptos, principios básicos y fundamentos matemáticos del SR, e incluye investigaciones y tendencias de él, destacando su desarrollo y sus retos en Colombia, y un caso de uso de HSI en la geología colombiana, cuyas evaluaciones muestran la capacidad de detección del sensor hiperespectral Hyperion, ubicado en el satélite EO-1, para el mapeo geológico en un sitio de prueba al noroccidente del municipio de Girón, departamento de Santander. Los resultados de las

\footnotetext{
* Universidad Industrial de Santander (Bucaramanga-Santander, Colombia). ariolfo.camacho@correo.uis.edu.co.

** M.Sc. Universidad Industrial de Santander (Bucaramanga-Santander, Colombia).

*** M.Sc. Universidad Autónoma de Bucaramanga (Bucaramanga-Santander, Colombia).

**** Ph.D. Universidad Politécnica De Madrid (Madrid, España).

****** Ph.D. Universidad De Delaware (Delaware, Estado Unidos).
} 
evaluaciones son satisfactorios; espectralmente, el coeficiente de correlación fue alto y la relación espacial entre la firma espectral obtenida y la geología conocida del área fue aceptable y correspondió al análisis de Difracción de Rayos X (DRX) realizado a muestras tomadas del área de estudio.

Palabras clave: Sensado Remoto, Imágenes Hiperespectrales, Firma espectral, Geología, Algoritmos de Detección de Objetivos.

\section{Abstract}

Remote Sensing (RS) is a data acquisition technique that requires no physical contact with the scene, through the use of sensors on aerial platforms. These sensors capture information in the electromagnetic spectrum different ranges, including the visible ones (VIS), the near infrared (NIR) and short wave range infrared (SWIR). Taking into account that each material present in a scene has different spectral characteristics, it is possible to analyze the spectral signatures and to use their identification and/or classification algorithms. Hyperspectral Image (HSI) remotely sensed in hundreds of spectral bands. HSI is important in different areas such as geology, mineralogy, agronomy, geography, environment, among others. However, the high volume of literature spread into different lines (RS, HSI and geology) makes it difficult to access and analyze it.

This paper presents a summary of concepts, principles, and mathematical foundations, including RS research and trends, highlighting its development and challenges in Colombia, and a HSI case use in the Colombian geology, which shows the detection capability of the Hyperion hyperspectral sensor, located in the EQ-1 Satellite for geological mapping, in a test site, at the Girón town northwest of the Santander, Colombia. The results of these evaluations are correlated with the geological information obtained by analysis of X-ray diffraction (XRD), performed on the samples taken from the studied area.

Keywords: Remote Sensing, Hyperspectral Imaging, Spectral Signature, Geology, Target Detection Algorithms.

\section{Resumo}

O Sensoriamento Remoto (SR) é uma técnica que permite captar informação de uma cena sem entrar em contato físico com ela, mediante o emprego de sensores localizados, principalmente, em plataformas aéreas, os quais captam informação em diferentes intervalos do espectro eletromagnético, incluindo o visível (VIS), o infravermelho próximo (NIR) e o de ondas curtas do infravermelho (SWIR). Tendo em conta que cada material presente em uma cena tem características espectrais diferentes, é possível, através da análise das assinaturas espectrais, realizar sua identificação ou classificação mediante algoritmos. As Imagens Hiperespectrais (HSI) captadas por sensores remotos em centenas de bandas espectrais são de importância em áreas como a geologia, a mineralogia, a agronomia e a ecologia, entre outras; porém, o grande volume de literatura dispersa em diferentes linhas (SR, HSI e geologia) dificulta seu acesso e análise. Este trabalho apresenta um compêndio de conceitos, princípios básicos e fundamentos matemáticos do SR, e inclui pesquisas e tendências dele, destacando seu desenvolvimento e seus desafios na Colômbia, e um caso de uso de HSI na geologia colombiana, cujas avaliações mostram a capacidade de detecção do sensor hiperespectral Hyperion, localizado no satélite EO-1, para o mapeamento geológico em um lugar de prova ao noroeste do município de Girón, Estado de Santander. Os resultados das avaliações são satisfatórios; espectralmente, o coeficiente de correlação foi alto e a relação espacial entre a assinatura espectral obtida e a geologia conhecida da área foi aceitável e correspondeu à análise de Difração de Raios X (DRX) realizada em amostras tomadas na área de estudo.

Palavras chave: Sensoriamento Remoto, Imagens Hiperespectrais, Assinatura espectral, Geologia, Algoritmos de Detecção de Objetivos. 


\section{INTRODUCCIÓN}

Los términos percepción remota, teledetección o sensado remoto hacen referencia, generalmente, al uso de tecnologías de sensores aéreos en la detección o clasificación de objetos en la Tierra (en la superficie, en la atmósfera y los océanos) a través del análisis de señales propagadas como la radiación electromagnética.

La técnica de Sensado Remoto (SR) permite adquirir información espacial, temporal y espectral de un objeto o escena sin entrar en contacto físico con él [1]. Los datos adquiridos van desde imágenes multiespectrales hasta imágenes hiperespectrales. Específicamente, el impacto de las imágenes hiperespectrales en la comunidad científica se refleja en el gran número de investigaciones [2-5] que involucran mejoras en su tecnología y aplicaciones en diversas disciplinas científicas, incluyendo la biología, la agronomía, la ecología, la oceanografía, la geología y la geografía, entre otras [6-10].

Gracias a la evolución electrónica y óptica de los sensores, actualmente se cuenta con plataformas aeroespaciales, aerotransportadas o terrestres que tienen sensores con resoluciones espectrales que van desde 11 bandas, como el sensor multiespectral Landsat 8 [11], hasta 220 bandas, como el sensor hiperespectral Hyperion [12], y resoluciones espaciales desde los $30 \mathrm{~cm}$ (Quickbird, Ikonos o WorldView-3) [13].

La fusión de técnicas como SR y espectroscopia para la observación de la Tierra ha permitido realizar análisis cuantitativos y cualitativos de grandes superficies. La proyección de una imagen espectral permite la extracción de información que no es posible obtener visualmente. Un sensor hiperespectral capta imágenes espaciales bidimensionales (2D) sobre numerosas longitudes de onda del espectro electromagnético de forma contigua y angosta, creando un tercer eje de información espectral $(\lambda)$. Cada imagen contiene información espectral en una longitud de onda determinada, conocida como banda espectral. Los sensores hiperespectrales miden la radiancia reflejada (reflectancia) en un gran número de longitudes de onda o bandas contiguas $(>100$ bandas), originando una alta resolución espectral.
Las bandas captadas son agrupadas para producir un cubo hiperespectral, cuyas dimensiones son la combinación de información espacial $(x, y)$ y espectral $(\lambda)$ de la escena. Captar imágenes en alta resolución espectral define el concepto de Imagen Hiperespectral (HSI). Las HSI en SR centran la atención en medidas espectrales de la región de reflectancia solar, entre los $400 \mathrm{~nm}$ y los $2.500 \mathrm{~nm}$, abarcando el espectro visible (VIS), el infrarrojo cercano (NIR) y las ondas cortas del infrarrojo (SWIR) [14]. La capacidad de los sensores HSI de adquirir mayor información espectral explota el hecho de que los materiales que comprenden los diversos objetos en una escena tienen un comportamiento espectral diferente de acuerdo con su composición [15]. Si se mide la radiación reflejada al sensor en las diferentes longitudes de onda sobre una región espectral suficientemente amplia, la firma espectral resultante puede utilizarse para detectar y discriminar diferentes materiales en una escena [14].

El uso de SR es de gran importancia en estudios geológicos. La cartografía geológica clásica y la exploración de minerales utilizan las características físicas de las rocas y los suelos, las firmas geoquímicas y los accidentes geográficos para determinar la naturaleza y la distribución de unidades geológicas e identificar los sitios de exploración de metales y minerales industriales. Diferencias sutiles mineralógicas son importantes para identificar formaciones de rocas o diferenciar áreas de tierra estéril de otras con un potencial económico minero; sin embargo, la identificación en campo presenta un alto grado de dificultad [16]. El uso de HSI a través de sensores remotos para captar información espacial y espectral en grandes extensiones de terrenos permite realizar mediciones de la superficie de la Tierra en cientos de imágenes espectrales, proporcionando un medio rápido de mapeo geológico y exploración minera. Actualmente, existe gran variedad de datos hiperespectrales, junto con algoritmos de clasificación y detección, que contribuyen al análisis cuantitativo de los datos y a la producción de mapas geológicos.

En la última década, el número de publicaciones, citaciones científicas [3] y aplicaciones de las técnicas de SR e HSI ha aumentado, principalmente en países desarrollados, en diferentes áreas, dentro de las cuales se destaca la geología; sin embargo, 
encontrar literatura científica de las dos técnicas de manera condensada, donde se evidencien sus principios básicos, aplicaciones, investigaciones, desarrollos $\mathrm{y}$, sobre todo, aplicaciones específicas en el área de geología en Colombia es difícil e implica la búsqueda en un gran número de fuentes dispersas e independientes entre SR, HSI, geología y sensores. Adicionalmente, la extensa oferta de imágenes captadas por múltiples plataformas que orbitan el planeta, y la diversidad de herramientas de software para su procesamiento [17] generan gran número de opciones que obligan al investigador o geólogo a realizar un análisis exhaustivo de la oferta existente, labor que requiere de tiempo, conocimientos y dominio de términos y conceptos de SR, HSI y su uso.

El artículo presenta un compendio de conceptos, principios físicos y matemáticos, investigaciones $\mathrm{y}$ aplicaciones que comprenden SR e HSI. Así mismo, describe el desarrollo y los retos de la aplicación de SR como herramienta de apoyo a la exploración y mapeo geológico en Colombia. Finalmente, se realiza una evaluación de la técnica de SR hiperespectral aplicada al área de la geología colombiana, a partir de la captura de imágenes del sensor hiperespectral Hyperion. El área de prueba para la evaluación está localizada en el municipio de Girón (Santander, Colombia).

\section{Sensado Remoto: desarrollo, PRINCIPIOS Y FUNDAMENTOS}

Sensado remoto (del inglés remote sensing) hace referencia a la técnica empleada para adquirir información sobre un objeto o fenómeno sin hacer contacto físico con él [14], a través de sensores ubicados en plataformas aerotransportadas (aviones tripulados o no tripulados - UAV) o aeroespaciales (satélites) [18]. También existe el SR en campo o laboratorio, donde el sensor es dirigido al objeto o escena in situ [19]. La Fig. 1 ilustra los elementos de un sistema de SR; el principal elemento es el sensor, que capta la energía electromagnética emitida desde una fuente de luz y que es reflejada por los elementos o materiales presentes en una escena. Los sensores pueden detectar o clasificar objetos, materiales, vegetación, minerales, tipo de suelos y rocas a través de señales propagadas (por ejemplo, radiación electromagnética).

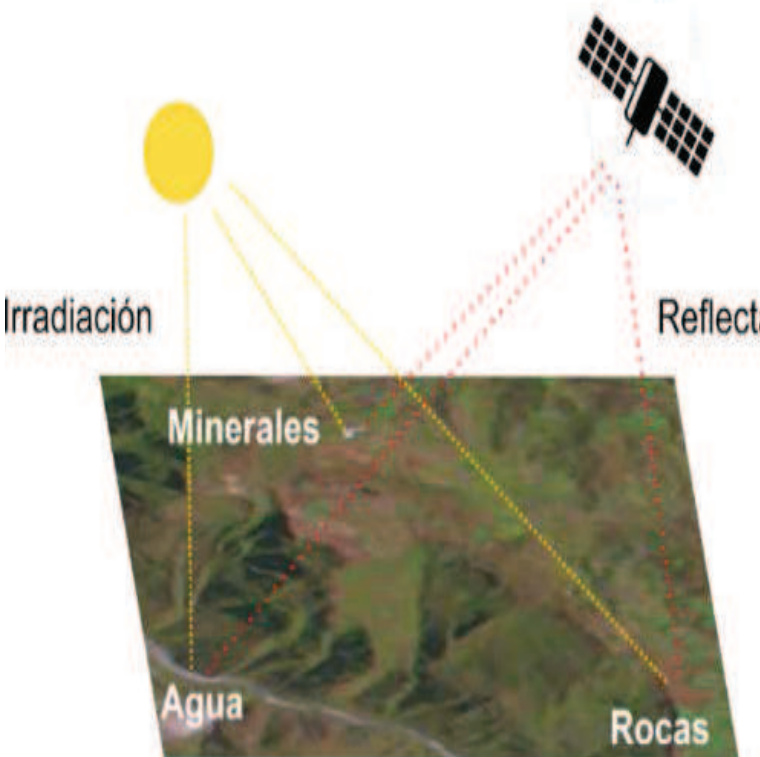

Fig. 1. Sistema de SR; se conforma por una fuente de energía, una escena, la trayectoria de la señal desde su origen hasta su destino y el sensor.

La historia de los sensores remotos se remonta a las primeras plataformas de observación aérea (globos aerostáticos) y a la invención de la fotografía, en el siglo XIX [20]. El SR moderno nace con la fotografía aérea, en el siglo XX [21], y su inicio en el espacio, en 1957, con el lanzamiento del satélite Sputnik; sin embargo, hasta 1969 se realizó la primera experiencia de fotografía orbital multiespectral para el estudio de los recursos terrestres [22], y en el año 2000 fue lanzado el satélite EO-1 (Earth Observing-1), primero en llevar un sensor hiperespectral (Hyperion) [22].

La evolución del SR ha sido marcada por tres hechos notables: 1) la aparición de la fotografía y el posterior desarrollo de sensores capaces de detectar radiación electromagnética en los rangos NIR y SWIR; 2) la transición de imágenes análogas a digitales [15, 23], y 3) la evolución de las telecomunicaciones, la cual permitió reducir los tiempos de transmisión de la información desde plataformas aeroespaciales.

De acuerdo con el informe de gestión 2012-2013 del Instituto Geográfico Agustín Codazzi (IGAC) [24], institución en Colombia que más utiliza y aplica $\mathrm{SR}$, principalmente en las áreas de la cartografía, la prevención y el control de desastres y el monitoreo meteorológico. El mayor número de sensores remotos con los cuales se obtienen imágenes de Colombia 
son multiespectrales; solo en casos muy especíicos, instituciones privadas solicitan al IGAC procesar o adquirir HSI [24].

\section{A. Fundamentos de imágenes espectrales}

Las condiciones de iluminación solar en una escena, así como las propiedades de reflexión de la luz en los materiales y las superficies hacen parte de las imágenes espectrales usadas en SR. La irradiancia se refiere a la energía de la luz por unidad tiempo (potencia) que incide sobre una superficie, normalizada por el área de superficie $\left(\mathrm{W} / \mathrm{m}^{2}\right)$. La reflectancia (luz reflejada) es un número adimensional entre 0 y 1 que caracteriza la fracción incidente de luz reflejada por una superficie captada por sensor [14].

Las características de reflexión de la luz solar de un material aportan a su detección y clasificación dentro de una escena. Cuando la irradiancia incide sobre un material, este absorbe $L_{\alpha}(\lambda)$, transmite $L_{\tau}(\lambda)$ y refleja $L_{\gamma}(\lambda)$, la luz de manera particular. Todos los componentes están en función de la longitud de onda.

$$
L_{i}(\lambda)=L_{\alpha}(\lambda)+L_{\tau}(\lambda)+L_{r}(\lambda)
$$

Las proporciones de en $L_{\alpha}(\lambda), L_{\tau}(\lambda)$ y $L_{r}(\lambda)$ (1) varían de acuerdo con el comportamiento espectral de los diferentes materiales encontrados dentro de la superficie de la Tierra. La variación espectral permite detectar o clasificar un objeto o material en una imagen captada por el sensor remoto.

La ecuación (2) permite cuantificar la reflectancia $\rho_{\lambda}$, midiendo la cantidad de irradiancia incidente $L_{i}(\lambda)$ que es reflejada, $L_{\gamma}(\lambda)$

$$
\rho_{\lambda}=\frac{L_{r}(\lambda)}{L_{i}(\lambda)}
$$

La variación de la reflectancia en función de la longitud de onda se denomina firma espectral, que es la medida cuantitativa de las propiedades espectrales de un objeto en el rango del espectro electromagnético captado por el sensor.

$\mathrm{Al}$ realizar el barrido sobre una superficie o escena, los sensores HSI detectan la energía solar reflejada por los diferentes materiales y miden la intensidad de la energía reflejada en diferentes longitudes de onda del espectro electromagnético (ver Fig. 2), creando las HSI, que son imágenes con información espacial $(x, y)$ y espectral $(\lambda)$, formando un hipercubo (3D). Cada píxel $(x, y)$ contiene un espectro de alta resolución espectral llamado firma espectral.

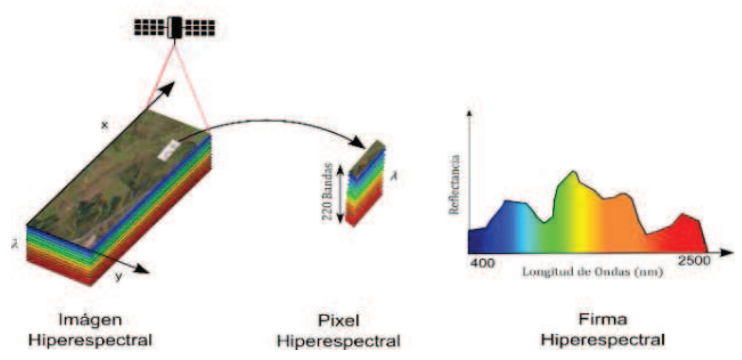

Fig. 2. Concepto de HSI y firma espectral. El sensor capta la energía solar reflejada por cada material presente en una escena espacial $(x, y)$ en diferentes longitudes de onda contiguas, creando un hipercubo. La firma espectral es la representación de un píxel $(\lambda)$.

\section{B. Imágenes multiespectrales versus imágenes hiperespectrales}

La resolución espectral se determina por el número de bandas espectrales y el ancho de ellas, usadas para medir la reflexión en diferentes longitudes de onda. La Fig. 3 muestra que las imágenes espectrales pueden clasificarse según el número de bandas que contengan: Las RGB o VIS, que pertenecen al rango visible del espectro; las multiespectrales, que capturan información en solo decenas de bandas, y las hiperespectrales, que contienen más de 100 bandas [25]. La principal fortaleza de las HSI sobre las imágenes multiespectrales es la gran cantidad de bandas contiguas espectrales posibles de adquirir y manejar, permitiendo la detección con mayor precisión de materiales en una escena.
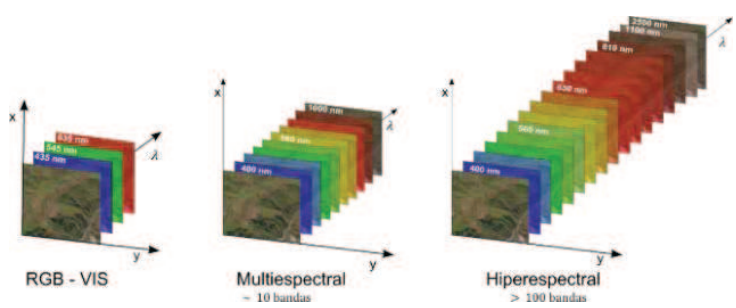

Fig. 3. Clasificación de las imágenes espectrales. El número de bandas y el ancho de estas determinan la resolución espectral de una imagen adquirida por los sensores remoto. 
Las imágenes multiespectrales facilitan la creación de mapas temáticos y de coberturas, y el uso de algoritmos de clasificación, pero los objetos solo pueden ser inferidos hasta cierto nivel de confianza, necesitando la comprobación de campo in situ [26]. Sin embargo, para detectar un material u objetivo en una escena, los resultados no son aceptables, comparados contra una HSI. Las HSI permiten la aplicación de algoritmos de detección de objetivos $[2,4,27]$ en datos de alta dimensionalidad a través de la firma espectral del objetivo por detectar (ej. un mineral). La firma espectral se puede obtener de librerías espectrales existentes, como la ASTER Version 2.0 [28], que contiene la librería espectral del Servicio Geológico de Estados Unidos (USGS) [29]; la librería espectral de la Universidad Johns Hopkins (JHU) [30], y la librería del laboratorio de la Nasa Jet Propulsion (JPL) [31].

La Fig. 4 ilustra la superioridad de las HSI sobre las imágenes multiespectrales. El mayor contenido de información en las imágenes HSI en función de la resolución espectral mejora y amplía su uso y aprovechamiento.

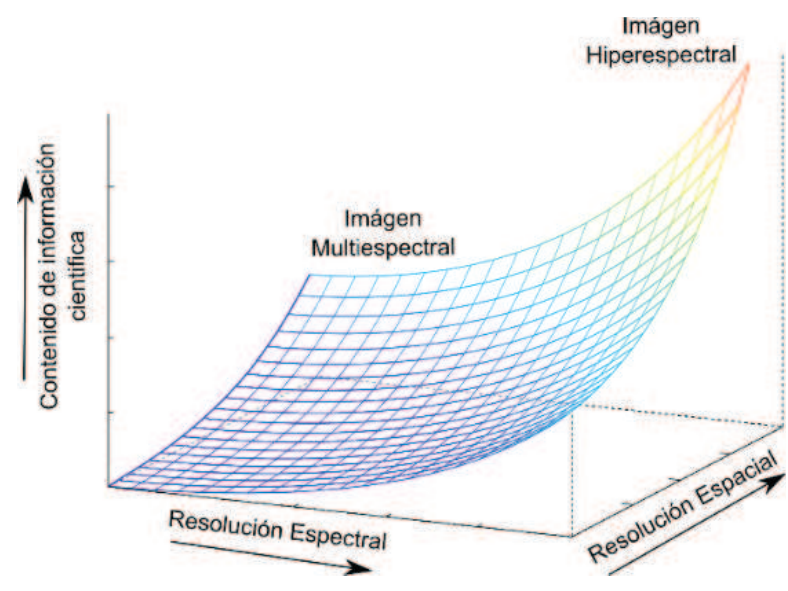

Fig. 4. Contenido de información y datos en una imagen de alta dimensionalidad. Modificado de [14].

\section{Sensores Remotos Hiperespectrales}

Los sensores remotos de HSI son instrumentos ópticos capaces de obtener datos a partir de la energía electromagnética reflejada de un objeto a distancia en cientos de bandas espectrales [32]. Los sensores pueden clasificarse como pasivos, si su fuente de energía es el Sol y captan su reflejo, y activos, si desde la misma plataforma donde se encuentra el sensor se emite la energía que después es medida por el sensor. Por otro lado, de acuerdo con la plataforma los sensores se pueden categorizar en sensores aerotransportados (aviones), aeroespaciales (satélites) o terrestres (cámaras hiperespectrales de campo).

Para evaluar la técnica de SR hiperespectral realizada en el presente estudio se usaron HSI captadas por el sensor Hyperion ubicado en la plataforma satelital EO-1 de la NASA. Hyperion es un sensor hiperespectral de alta resolución espectral, capaz de captar información espectral en 220 bandas, iniciando en la longitud de onda de los 355,59 nm hasta $2577,08 \mathrm{~nm}$, con un ancho de banda de $10 \mathrm{~nm}$ y resolución espacial de 30 m [33, 34]. Actualmente, Hyperion está bajo el control del Servicio Geológico de Estados Unidos (USGS) y la NASA. Hyperion capta escenas de la Tierra solo bajo solicitudes especificas, a través de la plataforma GeoBPMS [35].

\section{MÉTodo}

Para evaluar la capacidad de extraer información espectral de las HSI que pueden ser usadas y aplicadas en la geología colombiana, se solicitó al centro de vuelos espaciales Goddard, de la NASA, la adquisición de una imagen del departamento de Santander, Colombia; Hyperion adquirió el 13 de septiembre de 2014 una franja donde se ubica el lugar de estudio (ver Fig. 5-b).

\section{A. Lugar de estudio}

El área de prueba se localiza en el departamento de Santander (Colombia), específicamente en el municipio de Girón, entre los $7^{\circ} 02^{\prime} 51.79^{\prime \prime}$ y $6^{\circ} 58^{\prime}$ $13.39^{\prime}$ " de Latitud norte y los $73^{\circ} 15^{\prime} 33.30^{\prime \prime}$ y $73^{\circ}$ $10^{\prime} 53.63$ de Longitud al oeste de Greenwich. El departamento de Santander se encuentra localizado en una región tectónica compleja y en constante dinámismo, conocida como bloque Andes del Norte o bloque Norandino [36], lo que lo convierte en un área de interés para los geólogos. 


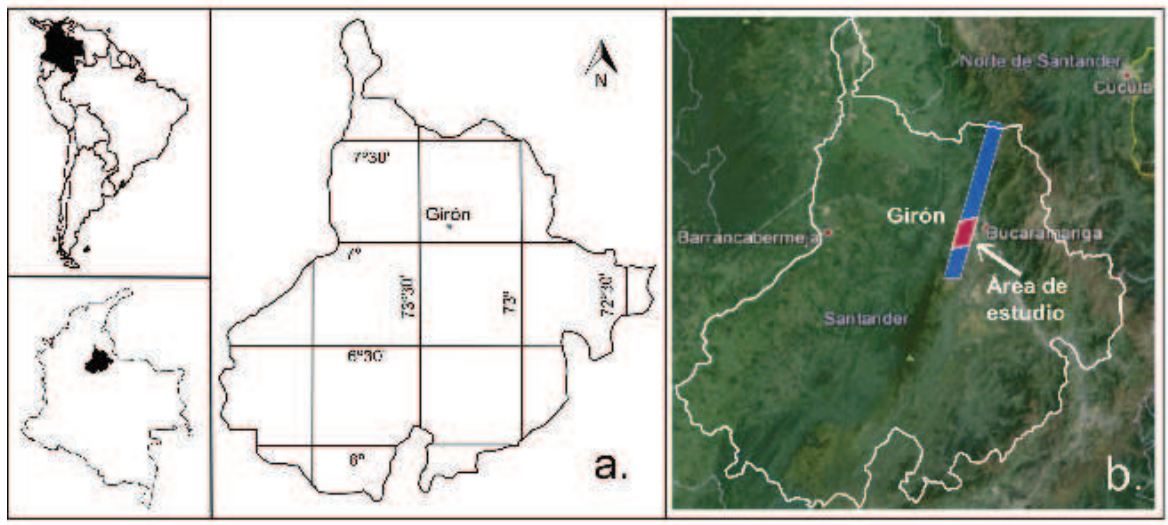

Fig. 5. Mapa de ubicación de el área de estudio. a) Ubicación del departamento de Santander. b) Ubicación específica del área de estudio al suroccidente del municipio de Girón.

Dentro del área de la imagen captada se encuentran las formaciones geológicas Kbeh y Kbal, señalada por el polígono rojo (ver Fig. 5-b), formaciones que son de interés para el presente estudio por sus condiciones de suelos desnudos y rojizos.

\section{B. Geología regional del área de estudio}

Desde el punto de vista geológico, en el área se ubican las unidades de las formaciones Kbeh y Kbal, localizadas en el municipio de Girón,
Santander, según registra el mapa geológico de Santander (ver Fig. 6). Las unidades corresponden al periodo cretácico inferior [36], y están formadas por sedimentitas, como areniscas, limolitas, calizas, lutitas, y lodolitas con niveles conglomeráticos y evaporíticos, que han sido datados del Berriasiano al Hauteriviano, cuyos afloramientos se encuentran en las estribaciones occidentales de la cordillera oriental. Estas sedimentitas están agrupadas en las formaciones Los Santos (Tambor), Cumbre y Rosa Blanca [36].

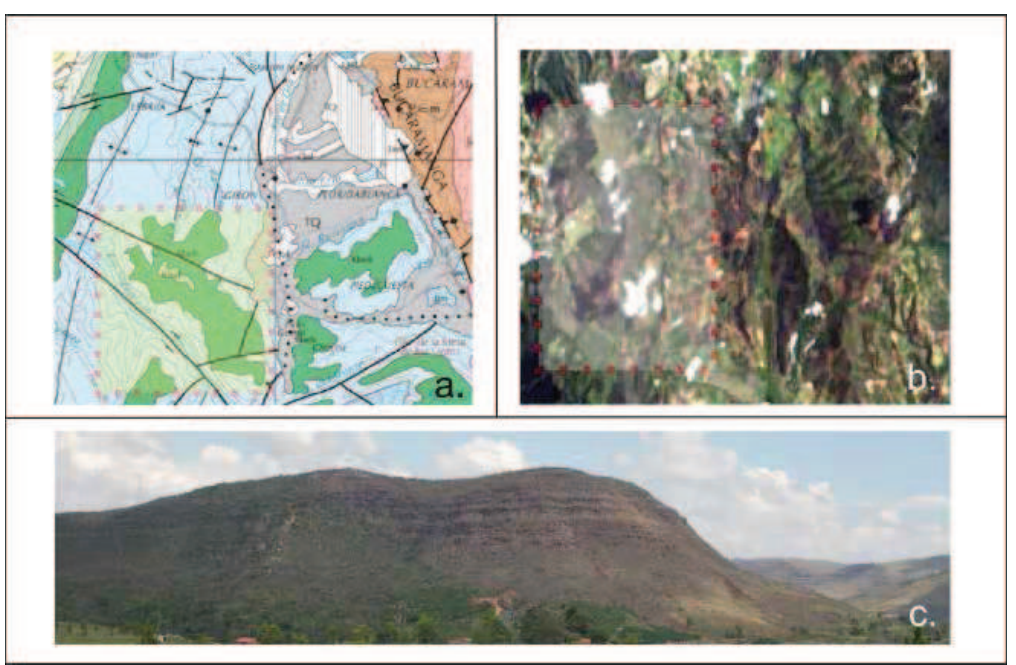

Fig. 6. Unidades geológicas Kbeh y Kbal. a) adaptación de mapa geológico de Santander 2007 (Ingeominas), b) vista satelital de Hyperion en color verdadero, c) fotografía área de estudio captada en salida de campo del 27 de septiembre de 2014. 


\section{Preprocesamiento de imagen hiperespectral}

Las HSI captadas presentan diferencias entre el valor de reflectancia registrado por el sensor frente a la reflectancia real, debido a factores como: el ángulo de azimut del sol, la altitud, las condiciones atmosféricas, la geometría de visión, la forma de cómo el sensor capta los datos, entre otras características del sensor [37]. Por lo tanto, se requiere realizar actividades de preprocesamiento, tales como: correcciones radiométricas, atmosféricas y eliminación de bandas con baja relación señal/ruido (SNR) antes de ser usadas en análisis o aplicación de algoritmos de clasificación o detección. La corrección radiométrica elimina el ruido por aparición de rayas (striping) [38]; la no calibración de los detectores crea rayas verticales donde los valores digitales de una banda son sistemáticamente inferiores a los de las columnas adyacentes. En el presente estudio se aplicó la herramienta de calibración radiométrica del software ENVI.

La corrección atmosférica es utilizada para compensar la pérdida de la señal, generada mientras esta viaja desde la fuente de iluminación hasta la escena, y es reflejada al sensor. Durante la corrección atmosférica son transformados los valores de radiancia en valores de reflectancia espectral [39]. Otro aspecto es validar las bandas con baja SNR o bandas malas (que contienen poco o nada de datos válidos). A través del análisis estadístico de las bandas y de encontrar los valores propios (eigen values) [33] de cada una, se ordenan de manera descendente, identificando las bandas con valores propios muy bajos (poca información valida) para ser retiradas.

\section{Resultados Y ANÁlisis}

Los resultados de la detección, clasificación y mapeo geológico de la escena de Hyperion se presentan en esta sección, centrándose en una porción del área de estudio, donde se ubican las formaciones Kbeh y Kbal (ver Figs. 5 y 6). De acuerdo con la geología del terreno y las coberturas vegetales, y combinando la información del mapa geológico de Santander [36], se extraen las firmas espectrales de la imagen, identificando los píxeles objetivos por analizar. De igual manera, se realizó una salida de campo con el objetivo de verificar la "verdad de terreno" (ground truth) y tomar muestras para su análisis en laboratorio. Parte de los píxeles en la escena no exhiben características espectrales correspondientes a minerales comerciales; existe sí una gran respuesta espectral de la vegetación verde presente en el área; sin embargo, después del preprocesamiento de la HSI y de la aplicación del algoritmo de detección espectral de Mapeo del Ángulo Espectral (SAM), que mide la similitud espectral entre dos muestras [40, 41], se detectaron espectros similares (a la firma espectral del mineral denominado nontronita, que es un mineral de la clase de los filosilicatos y pertenece al grupo de los minerales arcillosos llamado esmectita). La nontronita tiene distintas tonalidades de color. En este estudio, las tonalidades predominantes de la nontronita son de color rojizo, debido a la presencia de óxidos ferrosos en la superficie del área de estudio. La nontronita presenta características espectrales muy particulares que ayuda y facilita su identificación, como se muestra en la Fig. 7.

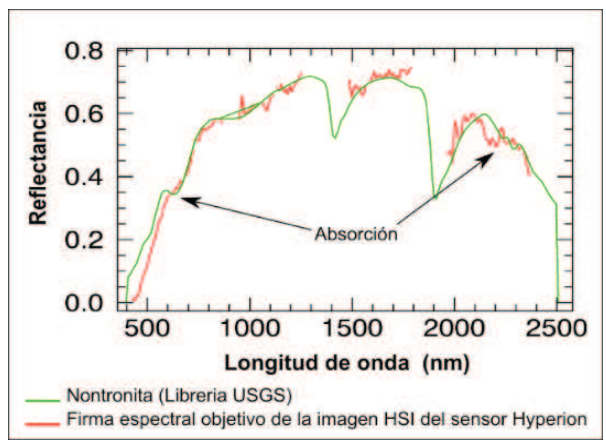

Fig. 7. Firma espectral de la nontronita frente a la firma identificada en la HSI, las firmas presentan absorción en $650 \mathrm{~nm}$ y $2200 \mathrm{~nm}$, característica comunes de presencia de óxidos ferrosos y arcillas en la nontronita.

Los datos de Hyperion (firma espectral objetivo) y la firma espectral de la nontronita (Librería USGS) presentan espectros de reflectancia muy similares; ambas curvas muestran absorción (caídas en la reflectancia) alrededor de $650 \mathrm{~nm}$ (óxidosferrosos) y en $2200 \mathrm{~nm}$ (minerales arcillosos), específicamente en el rango del SWIR las características se centran en $2200 \mathrm{~nm}, 2285 \mathrm{~nm}$ y $2300 \mathrm{~nm}[42,43]$.

Los resultados de detección sobre la HSI de Hyperion se ilustran en la Fig. 8, donde se mapea el área de estudio y se identifica la ubicación de superficies con presencia de nontronita. Las áreas que contienen mayor concentración están sobre las superficies de 
suelo desnudo, las cuales se pueden visualizar en la Fig. 8-d, por la proyección de la imagen en el rango SWIR, donde los tonos rosa representan suelos desnudos. En la cubierta superior de la colina (Fig. 6-c) se puede observar (Fig. 8-d) que existe mayor presencia de nontronita (esquina inferior izquierda de la misma figura).

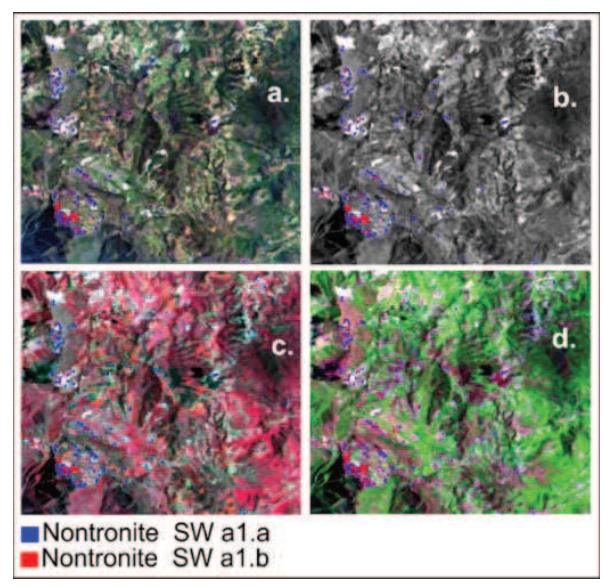

Fig. 8. Resultados del algoritmo de detección SAM en la HSI del área de estudio. El algoritmo SAM detecto en la HSI la presencia del mineral denominado nontronita a partir de dos firmas espectrales de la librería espectral USGS (nontronite SW a1.a y nontronite SW a1.b. a) Imagen en color verdadero, b) Banda 20 en escala de grises, c) Color falso que facilita el reconocimiento de las coberturas vegetales representado en tonos rojos, d) Imagen en el rango SWIR que ayuda a identificar superficies con suelo desnudo.

Los resultados alcanzados por el análisis de los datos obtenidos de las imágenes hiperespectrales se verifican a través de la verdad de terreno y el análisis de laboratorio, realizado mediante la técnica de difracción de rayos $\mathrm{X}$ (DRX), en tres muestras tomadas en terreno (HAF032, HAF033 y HAF2); los resultados cualitativos y cuantitativos (diafractogramas) corroboran la presencia de nontronita, y su composición química evidencia la presencia titanio, magnesio, óxidos de silicio y óxidos de hierro, comunes en las nontronitas. De igual manera, los resultados son equivalentes con el análisis de DRX de las firmas espectrales reportadas en la librería USGS (nontronite SW a1.a y nontronite SW a1.b).

\section{CONCLUSIONES Y RETOS DE LAS IMÁGENES HSI CAPTADAS POR SENSORES REMOTOS}

\section{A. Separación o desmezclado espectral y sensado compresivo}

El SR y, específicamente, los procesos tradicionales de adquisición de HSI se enfrentan a dos dificultades: la primera se refiere a la baja resolución espacial de los sensores y a la presencia de múltiples materiales en una escena; el mezclado espectral corresponde a la presencia de varios materiales en un mismo píxel; la técnica que aborda el problema de desmezclado espectral (spectral unmixing) [44, 45] consiste en determinar los tipos de materiales presentes y el porcentaje de ellos (abundancia) en cada uno de los píxeles de la imagen. La segunda dificultad se centra en la adquisión de las HSI; tradicionalmente, esta se basa en el criterio de muestreo de Nyquist [46], que implica un barrido punto tras punto o línea por línea de la escena, lo cual requiere tiempos excesivos de adquisición y genera grandes volúmenes de datos para su posterior reconstrucción; el reto se ha abordado mediante el uso de técnicas de sensado compresivo y la aplicación de códigos de apertura [47-48]. Un código de apertura es una máscara bidimensional que modula las proyecciones de una fuente antes de llegar al detector, es decir, permite o bloquea el paso de la radiación electromagnética en determinados puntos del espacio [49]. La mayor cantidad de aplicaciones de los códigos de apertura incluyen matrices uniformemente redundantes para la formación de imágenes sin lentes [50] y arquitecturas de compresión de HSI [51-53]. La tendencia en SR hiperespectal está enfocada en nuevos y mejores algoritmos de separación espectral (unmixing), procesamiento en paralelo [54], alto rendimiento computacional [55] y la aplicación de técnicas de sensado compresivo para reducir los volumenes de datos [56].

\section{B. Sensores Hiperespectrales}

Dentro de los grandes retos de la comunidad científica de sensores HSI se encuentran: mejor SNR, mayor precisión radiométrica, menores tamaños y costos de sensores y mayor resolución 
espectral. Estados Unidos dio la primera pauta con los sensores hiperespectrales Aviris y Hyperion; sin embargo, el número de países que desarrollan y usan sensores hiperespectrales está en constante crecimiento [57-58]. En el año 2014 se registraban a nivel mundial ocho instrumentos hiperespectrales: dos en plataformas aéreas (Hydicey Aviris) y seis en vehículos espaciales (Hyperion, Enmap, Prisma, Chris, Hyspiriy Iasi8), sin contar las nuevas soluciones de productos y servicios de SR hiperespectral existentes, en las cuales se destacan empresas líderes a nivel mundial, como Headwall, Terra Remote Sensing, Specim, Analytical Spectral Devices y SpecTir. Empresas que han logrado la reducción de tamaño y peso de los sensores, permitiendo abrir una nueva tendencia en el uso y aplicación de sensores HSI sobre plataformas aéreas no tripuladas (UAV), como el aibot X6 de Aibotix, y vant de la empresa Precisionhawk.

\section{Sensado Remoto Hiperespectral en Colombia}

Las imágenes hiperespectrales se han convertido en una técnica fundamental para la observación de la Tierra, y para satisfacer a nivel mundial la creciente demanda de mapeo a gran escala en diferentes áreas (recursos naturales, mineros, geológicos y agrícolas, entre otros); sin embargo, en Colombia se observa que el uso de SR a través de HSI se ha dado a nivel comercial y privado en sus primeros pasos. Empresas como Anglo Gold Ashanti (extractora de oro), con presencia en Colombia junto a Specim (compañía de SR hiperespectral), desarrollaron entre los años 2004 y 2008 una exploración minera y geológica de áreas de alta prospectividad de extracción de oro [59], aplicando técnicas de SR hiperespectral. Por otro lado, la Agencia Nacional de Hidrocarburos (ANH) incluye actualmente en sus procesos de exploración el uso de HSI para identificar áreas de alta prospectividad [60]. En el área de la academia y la investigación científica han surgido, en los últimos 5 años, iniciativas para la investigación y aplicación de la técnica de SR con HSI en el país, tales como: la identificación de cultivos ilícitos utilizando SR [61]; la implementación y creación de un centro regional de investigación e innovación en bioinformática y fotónica, de la Universidad del Valle del Cauca [62]; la creación, por el IGAC, de una librería de firmas espectrales colombianas [63]; así como estudios en diferentes grupos de investigación de universidades, como la Universidad Distrital [64], la Universidad del Valle, la Universidad de Manizales y la Universidad Industrial de Santander, que han mostrado su interés por la investigación y las aplicaciones de SR hiperespectrales en diferentes áreas (ambiental, forestal, geológica y agrícola).

Por último, el crecimiento de las empresas fabricantes de UAV, la reducción de precios de los sensores HSI y el creciente interés por las investigaciones científicas y la aplicación de SR hiperespectral a la geología y otras áreas en Colombia prevén prontas producciones científicas y aplicaciones.

\section{Agradecimientos}

Los autores agradecen la colaboración sobresaliente y la amabilidad del Dr. Stuard Frye, funcionario del centro de vuelos espaciales Goddard de la NASA, quien ha dado un apoyo permanente para captar las HSI sobre el territorio colombiano; de igual manera, los autores dan un reconocimiento al laboratorio de rayos $X$ del Parque Tecnológico Guatiguará, de la Universidad Industrial de Santander -UIS-, que dirige el Dr. José Antonio Henao, por su apoyo en los análisis de DRX sobre las muestras recolectadas, y agradecen a la Vicerrectoría de Investigación y Extensión de la UIS por apoyar esta investigación, registrada bajo el título del proyecto: "Extracción y separación de información espectral en imágenes obtenidas de forma remota usando muestreo compresivo y aperturas codificadas de color" (Código VIE 1804).

\section{REFERENCIAS}

[1] J. A. Richards y X. Jia, Remote Sensing Digital Image Analysis, New York, Berlin: SpringerVerlag, 2006.

[2] N. M. Nasrabadi, "Hyperspectral Target Detection", IEEE Signal Processing Magazine, vol. 31, no 1, pp. 34-44, 2014.

[3] W.-K. Ma, J. M. Bioucas-Dias, J. Chanussot y P. Gader, "Signal and image processing in hyperspectral remote sensing", IEEE Signal Processing Magazine, vol. 31, $\mathrm{n}^{\mathrm{o}}$ 1, pp. 22-23, 2014. 
[4] D. Manolakis, D. Marden y G. A.-. Shaw, "Hyperspectral Image Processing for Automatic Target Detection Applications", Lincoln Laboratory Journal, vol. 14, $\mathrm{n}^{\circ}$ 1, pp. 79-116, 2003.

[5] L. Homolová, Z. Malenovský, J. G. Clevers y G. Garcia, "Review of optical-based remote sensing for plant trait mapping", Ecological Complexity, vol. 15, no 1, pp. 1-16, 2013.

[6] E. Bastidas y J.A. Carbonell, "Caracterización espectral y mineralógica de los suelos del valle del río Cauca por espectroscopía visible e infrarroja (400-2.500 nm)", Agronomía Colombiana, vol. 28, n 2, pp. 291-301, 2010.

[7] D. F. Correa y E. Posada, "The social and economic benefits of Remote Sensing and Earth Observation Satellite Systems", Tecnologías geoespaciales al servicio del desarrollo territorial, vol. 49, pp. 15-26, 2013.

[8] M. Zhang, Z. Qin, X. Liu y S. L. Ustin, "Detection of stress in tomatoes induced by late blight disease in California, USA, using hyperspectral remote sensing", International Journal of Applied Earth Observation and Geoinformation, vol. 4, nº 4, p. 295-310, 2003.

[9] S. M. Arafat, M. A. Aboelghar y E. F. Ahmed, "Crop Discrimination Using Field Hyper Spectral Remotely Sensed Data", Advances in Remote Sensing, vol. 2, pp. 63-70, 2013.

[10] T. H. Kurz, S. J. Buckley y J. A. Howell, "Close range hyperspectral imaging integrated with terrestrial lidar scanning applied to rock characterization at centimetre scale", International Archives of the Photogrammetry, Remote Sensing and Spatial Information Sciences, vol. 39, nº 5, pp. 417-422, 2012.

[11] USGS. GEOLOGICAL SURVEY, "http://landsat.usgs.gov/index.php",U.S. GEOLOGICAL SURVEY, 07-05-2014. [En línea]. Disponible: http://landsat.usgs.gov/band_designations_landsat_satellites.php.

[12] Earth Observing 1, EO-1, "Earth Observing 1 (EO-1) / Sensor Hyperion", 13-12-2011. [En línea]. Disponible: http://eo1.usgs.gov/sensors/ hyperion. [Último acceso: 06-08-2014].

[13] Digital Globe, 07-05-2014. [En línea]. Disponible: http:/www.digitalglobe.com/es/ about-us/content-collection.
[14] G. A. Shaw y H.-H. Burker, "Spectral Imaging for Remote Sensing", Lincoln Laboratory Journal, vol. 14, nº 1, pp. 3-28, 2003.

[15] J. B. Campbell, Introduction to Remote Sensing, Edition Seven, New York: Guilford Press, 2007.

[16] F. Kruse, "Advances in Hyperspectral Remote Sensing for Geologic Mapping and Exploration", Proceedings 9th Australasian Remote Sensing Conference, Sydney, Australia, 1998.

[17] M. Labrador García, J. A. Évora Brondo y M. A. Pérez, Satélites de Teledetección para la Gestión del Territorio, Canarias, España: Consejería de Agricultura, Ganadería, Pesca y Aguas del Gobierno de Canaria, 2012.

[18] A. C. Watts, V. G. Ambrosia y E. A. Hinkley, "Unmanned Aircraft Systems in Remote Sensing and Scientific Research: Classification and Considerations of Use", Remote Sensing, vol. $4, n^{\circ} 1$, pp. 1671-1692, 2012.

[19] C. Chen, Remote sensing: models and methods for image processing, 3ra ed., New York, FL: Crc Press Taylor and Francis Group, 2006.

[20] F. Ritchin, After Photography, New York: W. W. Norton \& Company, 2008.

[21] W. C. Van Den Hoonaard, Map Wordlsa History of Women in Cartography, Ontario, Canada: Wilfrid Laurier University Press, 2013.

[22] NASA, "earthobservatory.nasa.gov/-Features/ RemoteSensing/", 2410 2014. [En línea]. Disponible: http://earthobservatory.nasa.gov/ Features/RemoteSensing/remote.php.

[23] C. Pohl y J. L. Van Genderen, "Multisensor image fusion in remote sensing: concepts, methods and applications", International Journal of Remote Sensing, vol. 19, nº5,pp. 823854, 1998.

[24] IGAC, "Informe 2012-2013 Instituto Geografico Agustin Codazzi, IGAC", Oficina de Difusión y Mercadeo de información, IGAC, Bogotá, 2013.

[25] E. Adam, O. Mutanga y D. Rugege, "Multispectral and hyperspectral remote sensing for identification and mapping of wetland vegetation: a review", Wetlands Ecology and Management, vol. 18, $\mathrm{n}^{\circ}$ 3, pp. 281-296, 2010.

[26] T. V. King y R. N. Clark, Verification of Remotely Sensed Data, in Remote Sensing for Site Characterization, Berlin: Springer, pp. 5961, 2000. 
[27] H. Kwon y N. M. Nasrabadi, "A comparative analysis of kernel subspace target detectors for hyperspectral imagery", EURASIP Journal on Advances Signal Process, Article ID 29250, 13 pages, 2007.

[28] A. M. Baldridge, S. J. Hook, C. I. Grove y G. Rivera, "The ASTER spectral library version 2.0", Remote Sensing of Environment, vol. 113, $\mathrm{n}^{\mathrm{o}}$ 4, pp. 711-715, 2009.

[29] U.S. Geological Survey (USGS), "U.S. Geological Survey (USGS) Libraries Program" 30-10-2014. [En línea]. Disponible: http:// library.usgs.gov/.

[30] J. W. Salisbury, L. S. Walter, N. Vergo y D. M. D'Aria, Infrared (2.1-25 micrometers) Spectra of Minerals: Johns Hopkins University, Maryland: The Johns Hopkins University Press, 1991.

[31] S. Hook, C. Grove y E. Paylor, "Laboratory reflectance spectra of 160 minerals, 0.4 to 2.5 Micrometers: JPL", JPL Publication, pp. 152153, 1992.

[32] R. A. Schowengerdt, Remote sensing: models and methods for image processing (3rd ed.), Burlington. USA: Academic Press, 2007.

[33] M. K. Griffin, S. M. Hsu, H.-h. K. Burke, S. M. Orloff y C. A. Upham, "Examples of EO-1 Hyperion Data Analysis", Lincoln Laboratory Journal, vol. 15, n² 2, pp. 271-298, 2005.

[34] U.S. Geological Survey (USGS), Hyperion Level $1 G$ (L1GST) Product Output Files Data Format Control Book (DFCB), USGS, Sioux Falls, South Dakota, USA, Disponible: https:// eo1.usgs.gov/documents/Hyperion L1G_EO1DFCB.v.1.pdf. 2006.

[35] NASA-Stuart Frye, "GeoBPMS", 14-04-2015. [En línea]. Disponible: http://eo1.gsfc.nasa.gov/ new/sensorWebExp/index.html.

[36] J. M. Royero y J. Clavijo, Mapa Geológico Generalizado del Departamento de Santander, Bogotá: Ingeominas, 2001.

[37] B. Datt, T. R. McVicar, T. G. Van Niel, D. L. Jupp y J. S. Pearlman, "Preprocessing EO-1 Hyperion Hyperspectral Data to Support the Application of Agricultural Indexes," IEEE Transactions On Geoscience And Remote Sensing, vol. 41, nº 6, pp. 1246-1259, 2003.

[38] Y. Smara, Z. Hamadache y S. Chouaf, "Preprocessing EO-1 Hyperion hyperspectral data applied to forests and vegetation classification", de ForestSAT conference 2014, Riva del Garda, Italia, 2014.

[39] Exelis, Inc, Atmospheric Correction Module: QUAC and FLAASH, 2009. [En línea]. Disponible: https://www.exelisvis.com/ portals/0/pdfs/envi/Flaash_Module.pdf.

[40] F. A. Kruse, A. B. Lefkoff y J. W. Boardman, "The spectral image processing system (SIPS) interactive visualization and analysis of imaging spectrometer data", Remote Sensing Environmental, vol. 44, n 2 , pp. 145-163, 1993.

[41] F. van der Meer, "The effectiveness of spectral similarity measures for the analysis of hyperspectral imagery", International Journal of Applied Earth Observation and Geoinformation, vol. 8, no 1, pp. 3-17, 2006.

[42] M. Mounkaila, Spectral and Mineralogical Properties of Potential Dust Sources on a Transect from the Bodélé Depression (central Sahara) to the Lake Chad in the Sahel, Vol. 78, Univ. Hohenheim, 2006.

[43] A. Chudnovsky, A. Kostinski, L. Herrmann, I. Koren, G. Nutesku y E. Ben-Dor, "Hyperspectral space borne imaging of dustladen flows: Anatomy of Saharan dust storm from the Bodélé Depression", Remote Sensing of Environment, vol. 115, n 1, pp. 1013-1024, 2011.

[44] J.M.N ascimento y J. M. Bioucas, "Vertex Component Analysis: A Fast Algorithm to Unmix Hyperspectral Data", IEEE Transactions On Geoscience And Remote Sensing, vol. 43, $\mathrm{n}^{\circ}$ 4, pp. 898-910, 2005.

[45] J. Boardman, F. Kruse y R. Green, "Mapping target signatures via partial unmixing of AVIRIS data", Fifth JPL Airborne Earth Science Workshop, vol. 95, $\mathrm{n}^{\circ}$ 1, pp. 23-26, 1995.

[46] A. J. Jerri, "The Shannon sampling theorem; Its various extensions and applications: A tutorial review", Proceedings of the IEEE, vol. 65, $\mathrm{n}^{\circ}$ 11, pp. 1565-1596, 1977.

[47] A. Ramirez, H. Arguello, G. R. Arce y B. M. Sadler, "Spectral Image Classification from Optimal Code-Aperture Compressive Measurements", IEEE Transactions on Geoscience and Remote Sensing, vol. 52, $\mathrm{n}^{\mathrm{o}} 6$, pp. 3299-3309, 2014.

[48] D. F. Galvis, Y. H. Mejía y H. Arguello, "Efficient reconstruction of Raman spectroscopy 
imaging based on compressive sensing", Dyna, vol. 81, nº 188, pp. 116-124, 2014.

[49] D. Brady, Optical Imaging and Spectroscopy, Durham, North Carolina, USA:Wyley, 2009.

[50] S. Gottesman y E. Fenimore, "New family of binary arrays for coded aperture imaging", Applied Optics, vol. 28, n 20, pp. 4344-4352, 1989.

[51] H. Arguello, H. Rueda, Y. Wu, W. Prather y G. Arce, "Higher-order computational model for coded aperture spectral imaging", Applied Optics, vol. 56, n ${ }^{\circ}$ 10, pp. D12-D21, 2013.

[52] G. Arce y H. Arguello, "Rank minimization code aperture design for spectrally", IEEE Trans. image Process, vol. 22, $\mathrm{n}^{\circ} 3$, pp. 941954, 2013.

[53] H. F. Rueda, A. Parada Mayorga y H. Arguello, "Spectral resolution enhancement of hyperspectral imagery by a multipleaperture compressive optical imaging system", Ingeniería e Investigación, vol. 34, n 3, pp. 5055, 2014.

[54] A. Plaza, J. Plaza, A. Paz y S. Sánchez, "Parallel Hyperspectral Image and Signal Processing", IEEE Signal Processing Mag, vol. 28, nº 3, pp. 119-126, 2011.

[55] A. J. Plaza y C.-I. Chang, High Performance Computing in Remote Sensing, New York: Chapman \& Hall, 2008.

[56] J. M. Bioucas-Dias, A. Plaza, G. Camps-Valls, P. Scheunders, N. M. Nasrabadi y J. Chanussot, "Hyperspectral Remote Sensing Data Analysis and Future Challenges", IEEE Geoscience and remote sensing magazine, vol. $1, \mathrm{n}^{\circ} 2$, pp. 6-36, 2013.

[57] Q. Tong, Y. Xue y L. Zhang, "Progress in Hyperspectral Remote Sensing Science and
Technology in China Over the Past Three Decades", IEEE Journal of Selected Topics in Applied Earth Observations and Remote Sensing, vol. 7, no 1,pp. 70-91, 2014.

[58] Sugianto, R. Merton y S. Laffan, "An Overview of the CHRIS/PROBA Mission: A New Generation of Multiangle Hyperspectral Remote Sensing and Its Application to Agriculture" $\mathrm{n}^{\circ} \mathrm{TS} 22.2$, New Development and Applications for Imagery Conference, Jakarta, Indonesia, 2004.

[59] AngloGold Ashanti, "Anglogold Ashanti Colombia", 28-01-2015. [En línea]. Disponible: http://www.anglogoldashanti.com.co/.

[60] ANH, Agencia Nacional de Hidrocarburos, "ANH", 01-02-2015. [En línea]. Disponible: http://www.anh.gov.co/buscar/Paginas/results. aspx?k=hiperespectral.

[61] Y. B. López, Metodología para identificar cultivos de coca mediante análisis de parámetros red edge y espectroscopia de imágenes, Tesis, Universidad Nacional de Colombia, Bogotá, 2012.

[62] Ministerio de Educación Colombia, "Centro Virtual de Noticias de la Educación", 04 02 2015. [En línea]. Disponible: http:// www.mineducacion.gov.co/cvn/1665/w3article-309017.html.

[63] ONU-SPIDER, "Oficina de Apoyo Regional de ONU-SPIDER", 2603 2014. [En línea]. Disponible: http://www.un-spider.org/es/ node/8292.

[64] I. Lizarazo, "Vegetation condition assessment using proximal and remote sensors", 2710 2013. [En línea]. Disponible: http://www. bdigital.unal.edu.co/46583/1/07795062.2014. pdf. 